PROCEEDINGS OF THE AMERICAN MATHEMATICAL SOCIETY

Volume 124, Number 11, November 1996

\title{
ON LIOUVILLE DECOMPOSITIONS IN LOCAL FIELDS
}

\author{
EDWARD B. BURGER
}

(Communicated by William W. Adams)

\begin{abstract}
In 1962 Erdős proved that every real number may be decomposed into a sum of Liouville numbers. Here we consider more general functions which decompose elements from an arbitrary local field into Liouville numbers. Several examples and applications are given. As an illustration, we prove that for any real numbers $\alpha_{1}, \alpha_{2}, \ldots, \alpha_{N}$, not equal to 0 or 1 , there exist uncountably many Liouville numbers $\sigma$ such that $\alpha_{1}^{\sigma}, \alpha_{2}^{\sigma}, \ldots, \alpha_{N}^{\sigma}$ are all Liouville numbers.
\end{abstract}

\section{INTRODUCTION}

A real number $\xi$ is a Liouville number if there exists an infinite sequence of rationals $p_{1} / q_{1}, p_{2} / q_{2}, \ldots$ so that

$$
\left|\xi-\frac{p_{n}}{q_{n}}\right|<\frac{1}{q_{n}{ }^{n}},
$$

for all $n=1,2, \ldots$ Thus, in the language of diophantine approximation, $\xi$ has excellent approximations by rationals of small height and this forces $\xi$ to be transcendental (see [12]). It turns out that the set of Liouville numbers has Lebesgue measure zero but is a dense $G_{\delta}$-set (see [9]). In 1962, Erdős [4] showed that every real number $\alpha$ may be expressed as the sum of two Liouville numbers. He gave both a constructive proof, where the Liouville numbers were explicitly given in base 2 as a function of the base 2 expansion of $\alpha$, and nonconstructive proof which followed from the properties of dense $G_{\delta}$-sets.

Recently this result has been extended to a broader range of the Mahler classification of numbers [7] (see [2]). In particular, Alniacik [1], using continued fraction techniques, proved that every real number, except possibly Liouville numbers, may be expressed as the sum of two $U_{2}$-numbers (recall that the set of $U_{1}$-numbers is precisely the set of Liouville numbers). Pollington [8], adopting methods of Schmidt [11], showed that for any natural number $n$, every real number may be decomposed as the sum of two $U_{n}$-numbers.

In this paper we wish to generalize Erdős' result in two different directions. Given $\alpha \in \mathbb{R}$, Erdős proved that there exist Liouville numbers $\sigma$ and $\tau$ so that $\alpha=f(\sigma, \tau)$, where $f(x, y)=x+y$. It is natural to wonder if $f(x, y)$ could be replaced by more interesting functions. In fact, Erdős himself showed that every nonzero real number is the product of two Liouville numbers, thus we may replace $f(x, y)$ above with $g(x, y)=x y$. Here we will extend this result to a very large class of functions.

Received by the editors April 6, 1994 and, in revised form, May 3, 1995.

1991 Mathematics Subject Classification. Primary 11J61, 11J81.

(C)1996 American Mathematical Society 
In another direction, we wish also to consider the analogous problem over an arbitrary local field. Let $K$ be a global field and $V_{K}$ denote the set of all nontrivial places of $K$. For each $w \in V_{K}$ we normalize an absolute value ||$_{w}$ so that the product formula holds: for $\gamma \in K, \gamma \neq 0, \prod_{w \in V_{K}}|\gamma|_{w}=1$. For $\gamma \in K$, we define the (inhomogeneous) height of $\gamma, h(\gamma)$, by

$$
h(\gamma)=\prod_{w \in V_{K}} \max \left\{1,|\gamma|_{w}\right\}
$$

We write $K_{w}$ for the completion of $K$ with respect to the place $w$.

For a fixed place $w \in V_{K}$, we say $\xi \in K_{w}$ is a Liouville number in $K_{w}$ if there exists an infinite sequence $\left\{\gamma_{n}\right\}_{n=1}^{\infty}$ in $K$ such that

$$
\left|\xi-\gamma_{n}\right|_{w}<\frac{1}{h\left(\gamma_{n}\right)^{n}},
$$

for all $n=1,2,3, \ldots$ It follows that if $\xi \in K_{w}$ is a Liouville number, then it is transcendental over $K$.

If $\mathcal{Z}_{w} \subseteq K_{w} \times K_{w}$ is an open subset, $f: \mathcal{Z}_{w} \rightarrow K_{w}$ a continuous function and $\alpha \in K_{w}$, then we say that $f$ is locally injective at $\alpha$ if there exist open sets $\mathcal{U}$ and $\mathcal{V}$ in $K_{w}, \mathcal{U} \times \mathcal{V} \subseteq \mathcal{Z}_{w}$, so that:

(i) for every $x \in \mathcal{U}$, there exists a unique $y \in \mathcal{V}$ so that $f(x, y)=\alpha$,

(ii) for every $y \in \mathcal{V}$, there exists a unique $x \in \mathcal{U}$ so that $f(x, y)=\alpha$. More precisely we say that $f$ is locally injective at $\alpha$ on $\mathcal{U} \times \mathcal{V}$.

We now state our first decomposition result.

Theorem 1. Let $w$ be a place of the global field $K, \mathcal{Z}_{w} \subseteq K_{w} \times K_{w}$ an open set, $f: \mathcal{Z}_{w} \rightarrow K_{w}$ a continuous function and $\alpha \in K_{w}$. If $f$ is locally injective at $\alpha$, then there exist Liouville numbers $\sigma$ and $\tau$ in $K_{w}$ such that

$$
f(\sigma, \tau)=\alpha .
$$

Before proving Theorem 1, we consider various examples and applications. We also indicate how one may produce a generalization of Theorem 1 which allows simultaneous decompositions with respect to several functions.

\section{LiOUVILLE DECOMPOSITIONS}

We begin by providing a natural class of functions that are locally injective. In particular, we show that there is no analogue of the Gelfond-Schneider Theorem for Liouville numbers. This requires us to formulate a generalized Implicit Function Theorem over arbitrary local fields. Such a result appears difficult to locate in the literature and thus we state it explicitly here. Suppose that f : $K_{w}^{M} \times K_{w}^{N} \rightarrow K_{w}^{N}$. We view the image of $\mathbf{f}$ as a row vector, $\mathbf{f}=\left(f_{1}, f_{2}, \ldots, f_{N}\right)$, where $f_{n}: K_{w}^{M} \times K_{w}^{N} \rightarrow$ $K_{w}$ for each $n=1,2, \ldots, N$. Also we view elements of $K_{w}^{M} \times K_{w}^{N}$ as $(\mathbf{x}, \mathbf{y})$, where $\mathbf{x}=\left(x_{1}, x_{2}, \ldots, x_{M}\right) \in K_{w}^{M}$ and $\mathbf{y}=\left(y_{1}, y_{2}, \ldots, y_{N}\right) \in K_{w}^{N}$.

Theorem 2 (The Implicit Function Theorem). Let $w$ be a place of the global field $K$ and $\mathcal{U}$ be an open set in $K_{w}^{M} \times K_{w}^{N}$. Suppose that $\mathbf{f}: \mathcal{U} \rightarrow K_{w}^{N}$ is a continuously differentiable function such that $\mathbf{f}\left(\mathbf{x}_{0}, \mathbf{y}_{0}\right)=\mathbf{0}$ for some $\left(\mathbf{x}_{0}, \mathbf{y}_{0}\right) \in \mathcal{U}$ and

$$
\operatorname{det}\left(\left(\frac{\partial f_{m}}{\partial y_{n}}\left(\mathbf{x}_{0}, \mathbf{y}_{0}\right)\right)_{m=1,2, \ldots, N}^{n=1,2, \ldots, N}\right) \neq 0 \text {. }
$$


Then there exist open sets $\mathcal{V} \subseteq K_{w}^{M} \times K_{w}^{N}$ and $\mathcal{W} \subseteq K_{w}^{M}$ with $\left(\mathbf{x}_{0}, \mathbf{y}_{0}\right) \in \mathcal{V}, \mathbf{x}_{0} \in \mathcal{W}$, and a continuously differentiable function $\mathbf{g}: \mathcal{W} \rightarrow K_{w}^{N}$ such that $(\mathbf{x}, \mathbf{g}(\mathbf{x})) \in \mathcal{V}$ and

$$
\mathbf{f}(\mathbf{x}, \mathbf{g}(\mathbf{x}))=\mathbf{0}
$$

for all $\mathbf{x} \in \mathcal{W}$.

The proof of Theorem 2 follows directly from the proof of the traditional (archimedean) Implicit Function Theorem (see [5], Section 4.6) together with the local field analogue of the Inverse Function Theorem (see [3], Section 1.5).

Suppose now that $f: \mathcal{Z}_{w} \rightarrow K_{w}$ is a continuously differentiable function with $f\left(x_{0}, y_{0}\right)=\alpha$, where $\left(x_{0}, y_{0}\right) \in \mathcal{Z}_{w}$. If $\frac{\partial f}{\partial y}\left(x_{0}, y_{0}\right) \neq 0$, then by Theorem 2 , there exists an open neighorhood $\mathcal{W}$ of $x_{0}$ and a continuously differentiable function $g: \mathcal{W} \rightarrow K_{w}$ so that $g\left(x_{0}\right)=y_{0}$ and $f(x, g(x))=\alpha$ for all $x \in \mathcal{W}$. If $g^{\prime}\left(x_{0}\right) \neq 0$, then $g$ is injective on some neighborhood of $x_{0}$ (see [10], Proposition 27.3) and thus it follows that $f$ is locally injective at $\alpha$. Hence any rational function $f(x, y) \in$ $K_{w}(x, y)$ will satisfy the hypotheses of Theorem 1 for suitable choices of $\alpha$.

As a basic illustration, if we select $f(x, y)=x+y$, then for any $\alpha \in K_{w}, f$ is locally injective at $\alpha$, thus we produce the Erdös result over $K_{w}$. As another example, suppose that $K=\mathbb{Q}$ and $p$ is a place of $\mathbb{Q}$. We now define the function $f_{p}(x, y)$ by

$$
f_{p}(x, y)=\frac{\log _{p}(1+x)}{y}=\frac{1}{y} \sum_{n=1}^{\infty} \frac{(-1)^{n+1} x^{n}}{n},
$$

and let $\alpha_{p} \in \mathbb{Q}_{p} \backslash\{0\}$. For $p$ a nonarchimedean place, if we consider the open sets

$$
\mathcal{U}_{p}=\left\{x \in \mathbb{Q}_{p}:|x|_{p}<p^{-1 /(p-1)}\right\}
$$

and

$$
\mathcal{V}_{p}=\left\{y \in \mathbb{Q}_{p}: 0<|y|_{p}<\left|\alpha_{p}\right|_{p}^{-1} p^{-1 /(p-1)}\right\},
$$

then it follows that $f_{p}(x, y)$ is locally injective at $\alpha_{p}$ on $\mathcal{U}_{p} \times \mathcal{V}_{p}$. For $p=\infty$, we may just take $\mathcal{U}_{\infty}=\left\{x \in \mathbb{Q}_{\infty}: x>-1\right\}$ and $\mathcal{V}_{\infty}=\mathbb{R}$ and conclude that $f_{\infty}(x, y)$ is locally injective on $\mathcal{U}_{\infty} \times \mathcal{V}_{\infty}$. Hence as a consequence of Theorem 1 , there exist two Liouville numbers $\sigma$ and $\tau$ in $\mathbb{Q}_{p}$ such that

$$
\exp _{p}\left(\alpha_{p} \tau_{p}\right)=\sigma_{p},
$$

where $\exp _{p}(x)=\sum_{n=0}^{\infty} x^{n} / n$ ! in $\mathbb{Q}_{p}$ (for a further discussion of $\exp _{p}$ and $\log _{p}$ for finite $p$, see [6]). In particular, given $\alpha_{p} \in \mathbb{Q}_{p}, \alpha_{p} \neq 0$, there exists a Liouville number $\tau_{p} \in \mathbb{Q}_{p}$ so that $\exp _{p}\left(\alpha_{p} \tau_{p}\right)$ is also a Liouville number in $\mathbb{Q}_{p}$.

As a final application, we recall the celebrated Gelfond-Schneider Theorem which states that for algebraic numbers $\mu$ and $\nu, \mu \neq 0, \mu \neq 1, \nu \notin \mathbb{Q}$, it follows that $\mu^{\nu}$ is transcendental. Now suppose that $\alpha$ is a positive real number not equal to 1. Then $f(x, y)=y^{x}$ is locally injective at $\alpha$ and thus by Theorem 1 there exist Liouville numbers $\sigma$ and $\tau$ in $\mathbb{R}$ such that

$$
\sigma^{\tau}=\alpha .
$$

Hence we conclude that there is no analogue of the Gelfond-Schneider Theorem for Liouville numbers.

For Theorem 1, our method of proof is to successively define two convergent sequences in $K$ concurrently: $s_{1}, t_{1}, s_{2}, t_{2}, \ldots$, in sympathy with the function $f$, 
such that the convergence of both sequences is so fast that they converge to Liouville numbers in $K_{w}$ and, by the construction, the Liouville numbers satisfy the theorem. As an immediate consequence of the proof, we may deduce the following simultaneous decomposition generalization of Theorem 1.

Theorem 3. Let $w$ be a place of the global field $K ; \mathcal{U}, \mathcal{V}_{1}, \mathcal{V}_{2}, \ldots, \mathcal{V}_{N}$ open sets in $K_{w}$ and $\alpha_{1}, \alpha_{2}, \ldots, \alpha_{N}$ elements in $K_{w}$. If for each $n, 1 \leq n \leq N, f_{n}$ : $\mathcal{U} \times \mathcal{V}_{n} \rightarrow K_{w}$ is locally injective at $\alpha_{n}$ on $\mathcal{U} \times \mathcal{V}_{n}$, then there exist Liouville numbers $\sigma, \tau_{1}, \tau_{2}, \ldots, \tau_{N}$ in $K_{w}$ so that

$$
f_{n}\left(\sigma, \tau_{n}\right)=\alpha_{n}
$$

for all $n=1,2, \ldots, N$.

As an example in the case of $K_{w}=\mathbb{R}$, let $\alpha_{1}, \alpha_{2}, \ldots, \alpha_{N}$ be real numbers not equal to 0 or 1 . Then plainly the function $f_{n}(x, y)=y^{1 / x}$ is locally injective at $\alpha_{n}$. Thus by Theorem 3, there exists a Liouville number $\sigma$ such that

$$
\alpha_{1}^{\sigma}, \alpha_{2}^{\sigma}, \ldots, \alpha_{N}^{\sigma}
$$

are all Liouville numbers. In particular, there exists a Liouville number $\tau$ such that $\log \tau$ is also a Liouville number. Various such examples may be constructed from other well-known locally injective functions.

\section{The Proof of Theorem 1}

For $\gamma \in K_{w}$ and $\varepsilon>0$, we define the basic open set $B(\gamma, \varepsilon)$ by

$$
B(\gamma, \varepsilon)=\left\{x \in K_{w}:|x-\gamma|_{w}<\varepsilon\right\} .
$$

As $f$ is locally injective at $\alpha$, there exist basic open sets $\mathcal{U}_{1}$ and $\mathcal{V}_{1}$ in $K_{w}$ such that $f$ is locally injective at $\alpha$ on $\mathcal{U}_{1} \times \mathcal{V}_{1}$. Since $K$ is a dense subset of $K_{w}$, we may select an element $s_{1} \in K$ so that $s_{1} \in \mathcal{U}_{1}$. We now let $\varepsilon_{1}>0$ be so small that if we define $\mathcal{W}_{1}=B\left(s_{1}, \varepsilon_{1}\right)$, then $\mathcal{W}_{1} \subseteq \mathcal{U}_{1}$ and $\varepsilon_{1} \leq h\left(s_{1}\right)^{-1}$. Next, let $\mathcal{Y}_{1} \subseteq \mathcal{V}_{1}$ be a basic open set in $K_{w}$ so that $f$ is locally injective on $\mathcal{W}_{1} \times \mathcal{Y}_{1}$ and select a $t_{1} \in K$ so that $t_{1} \in \mathcal{Y}_{1}$ and $f\left(u_{1}, t_{1}\right)=\alpha$ for some $u_{1} \in \mathcal{W}_{1}$. We now let $\delta_{1}>0$ be so small that if we define $\mathcal{V}_{2}=B\left(t_{1}, \delta_{1}\right)$, then $\mathcal{V}_{2} \subseteq \mathcal{Y}_{1}$ and $\delta_{1} \leq h\left(t_{1}\right)^{-1}$. Finally, we let $\mathcal{U}_{2}$ be a basic open set in $K_{w}$ such that $u_{1} \in \mathcal{U}_{2} \subseteq \mathcal{W}_{1}$ and $f$ is locally injective at $\alpha$ on $\mathcal{U}_{2} \times \mathcal{V}_{2}$.

Assuming that $\left\{s_{1}, s_{2}, \ldots, s_{N-1}\right\} \subseteq K,\left\{t_{1}, t_{2}, \ldots, t_{N-1}\right\} \subseteq K$ and basic open sets $\mathcal{U}_{N}, \mathcal{V}_{N}$ in $K_{w}$, with $f$ locally injective at $\alpha$ on $\mathcal{U}_{N} \times \mathcal{V}_{N}$, have all been defined, we now construct $s_{N}$ and $t_{N}$. Let $s_{N}$ be a point of $K$ such that $s_{N} \in \mathcal{U}_{N}, s_{N} \notin$ $\left\{s_{1}, s_{2}, \ldots, s_{N-1}\right\}$. We select $\varepsilon_{N}>0$ so small that if we define $\mathcal{W}_{N}=B\left(s_{N}, \varepsilon_{N}\right)$, then $\mathcal{W}_{N} \subseteq \mathcal{U}_{N}$ and $\varepsilon_{N} \leq h\left(s_{N}\right)^{-N}$. Let $\mathcal{Y}_{N} \subseteq \mathcal{V}_{N}$ be a basic open set in $K_{w}$ so that $f$ is locally injective on $\mathcal{W}_{N} \times \mathcal{Y}_{N}$ and select an element $t_{N} \in K$ such that $t_{N} \in \mathcal{Y}_{N}, t_{N} \notin\left\{t_{1}, t_{2}, \ldots, t_{N-1}\right\}$ and $f\left(u_{N}, t_{N}\right)=\alpha$ for some $u_{N} \in \mathcal{W}_{N}$. Next we select $\delta_{N}>0$ so small that if we define $\mathcal{V}_{N+1}=B\left(t_{N}, \delta_{N}\right)$, then $\mathcal{V}_{N+1} \subseteq \mathcal{Y}_{N}$ and $\delta_{N} \leq h\left(t_{N}\right)^{-N}$. Finally we choose a basic open set $\mathcal{U}_{N+1} \subseteq K_{w}$ so that $u_{N} \in \mathcal{U}_{N+1} \subseteq \mathcal{W}_{N}$ and $f$ is locally injective at $\alpha$ on $\mathcal{U}_{N+1} \times \mathcal{V}_{N+1}$.

We now observe that given any integer $M \geq 1$, for all sufficiently large integers $m_{1}, m_{2}$,

$$
\left|s_{m_{1}}-s_{m_{2}}\right|_{w}<\varepsilon_{M} \quad \text { and } \quad\left|t_{m_{1}}-t_{m_{2}}\right|_{w}<\delta_{M}
$$


Since both $\varepsilon_{M} \rightarrow 0$ and $\delta_{M} \rightarrow 0$ as $M \rightarrow \infty$, the sequences $\left\{s_{m}\right\}$ and $\left\{t_{m}\right\}$ converge in $K_{w}$. Let

$$
\lim _{m \rightarrow \infty} s_{m}=\sigma \quad \text { and } \quad \lim _{m \rightarrow \infty} t_{m}=\tau \text { in } K_{w} .
$$

We also remark that from our construction we have

$$
\lim _{m \rightarrow \infty} u_{m}=\sigma \quad \text { in } K_{w} .
$$

By our upper bounds on $\varepsilon_{m}$ and $\delta_{m}$ we conclude that for all sufficiently large $m$,

$$
\left|\sigma-s_{m}\right|_{w}<h\left(s_{m}\right)^{-m} \text { and }\left|\tau-t_{m}\right|_{w}<h\left(t_{m}\right)^{-m} .
$$

Thus, both $\sigma$ and $\tau$ are Liouville numbers in $K_{w}$. Finally we recall that for all $m$,

$$
f\left(u_{m}, t_{m}\right)=\alpha .
$$

Hence the continuity of $f$ and the limit in (3.1) yield

$$
f(\sigma, \tau)=\alpha,
$$

which completes the proof.

\section{Concluding Remarks}

Of course the previous argument may be generalized to allow functions of more than two variables. In view of the Weak Approximation Theorem (see [13], Corollary 2, Chapter IV, Section 2) together with a Cantor diagonalization argument to introduce a new place of $K$ at each stage, one may select the sequences $\left\{s_{n}\right\}_{n=1}^{\infty}$ and $\left\{t_{n}\right\}_{n=1}^{\infty}$ so that they converge very rapidly at all places of $K$. Thus one may produce a "global" version of Theorem 1 where the pair of sequences $\left\{s_{n}\right\}_{n=1}^{\infty}$ and $\left\{t_{n}\right\}_{n=1}^{\infty}$ converge to Liouville numbers in all completions of $K$ and for each completion $K_{w}$, the Liouville numbers form a decomposition with respect to the locally injective function $f_{w}$ at $\alpha_{w} \in K_{w}$. Thus, in some sense, one may produce different Liouville decompositions simultaneously at all places. We note that one may recast such results to very general topological spaces having a height function and countably many metrics which are independent in the sense that an analogue of the Weak Approximation Theorem holds. For example, one could produce a version involving affine varieties. We also observe that in view of the product formula over $K$, it is impossible for such sequences to converge uniformly with respect to the various places of $K$.

Finally we remark that it would be interesting to produce an analogue of Theorem 1, where the conclusion that $\sigma$ and $\tau$ are Liouville numbers is replaced by the conclusion that $\sigma$ and $\tau$ are $U_{n}$-numbers for a particular $n>1$. It may be possible to modify the argument of Pollington [8] to produce such a result in the case when $K=\mathbb{Q}$.

\section{ACKNOWLEDGMent}

The author wishes to thank Professors J.D. Vaaler, D. Witte and the referee for their helpful remarks regarding this paper. 


\section{REFERENCES}

1. K. Alniacik, Representation of real numbers as sums of $U_{2}$-numbers, Acta Arith. 55 (1990), 301-310. MR 91i: 11077

2. A. Baker, Transcendental Number Theory, Cambridge University Press, Cambridge and London, 1975. MR 54:10163

3. N. Bourbaki, Variétés différentielles et analytiques, Éléments de Mathématique XXXIII, Hermann, Paris, 1967. MR 36:2161

4. P. Erdős, Representations of real numbers as sums and products of Liouville numbers, Michigan Math. J. 9 (1962), 59-60. MR 24:A3134

5. W. Fleming, Functions of Several Variables (2nd Edition), Springer-Verlag, New York, 1977. MR 54:10514

6. N. Koblitz, p-adic Numbers, p-adic Analysis, and Zeta-Functions (Second Edition), SpringerVerlag, New York, 1984. MR 86c:11086

7. K. Mahler, Zur Approximation der Exponentialfunktion und del Logarithmus, I, J. Reine Angew. Math. 166 (1932), 118-136.

8. A.D. Pollington, Sum Sets and U-numbers, Number Theory with an Emphasis on the Markoff Spectrum (A. Pollington and W. Moran, eds.), Lecture Notes in Pure and Applied Mathematics 147, Marcel Dekker, New York, 1993. MR 94d:11051

9. W.M. Priestley, Sets Thick and Thin, M.A.A. Monthly 83 (1976), 648-650. MR 54:5127

10. W.H. Schikhof, Ultrametric calculus, Cambridge studies in advanced matehmatics 4, Cambridge University Press, Cambridge and London, 1984. MR 86j:11104

11. W.M. Schmidt, On badly approximable numbers and certain games, Trans. Amer. Math. Soc. 123 (1966), 178-199. MR 33:3793

12. W.M. Schmidt, Diophantine Approximation, Springer Lecture Notes 785, Springer-Verlag, Berlin-Heidelberg-New York, 1980. MR 81j:10038

13. A. Weil, Basic Number Theory, Springer-Verlag, Berlin-Heidelberg-New York, 1974. MR 55:302

Department of Mathematics, Williams College, Williamstown, Massachusetts 01267

E-mail address: Edward.B.Burger@williams.edu 